\title{
Hand posture and cognitive control: The congruency sequence effect is reduced near the hands
}

\author{
Julia Englert $^{1} \cdot$ Dirk Wentura $^{1}$
}

Published online: 19 January 2016

(C) Psychonomic Society, Inc. 2016

\begin{abstract}
According to several recent articles, attentional processing seems to be modulated by the proximity of one's own hand to a stimulus. Weidler and Abrams (Psychonomic Bulletin \& Review, 21, 462-469, 2014) found a significant reduction of the Eriksen flanker effect when the stimuli were presented close to the participants' hands. They interpreted this as evidence for stronger cognitive control near the hands. Using a near-by hands manipulation intended to vary distance while keeping posture of the hands constant, we found a modulation of the congruency sequence effect (CSE or Gratton effect), i.e., a larger flanker effect following incompatible trials than following compatible trials. The CSE was eliminated near the hands. Though we did not find a reduction in the flanker effect itself (which might be the result of using a near-by hand manipulation somewhat different from that of Weidler and Abrams), this result can be considered to be compatible with Weidler and Abrams' original hypothesis if the congruency sequence effect is interpreted in terms of cognitive control.
\end{abstract}

Keywords Hand posture - Cognitive and attentional control · Visual selective attention $\cdot$ Embodied cognition

\section{Introduction}

The space near the hands appears to receive privileged attentional processing (Brockmole, Davoli, Abrams, \& Witt,

Julia Englert

julia.englert@uni-saarland.de

1 Department of Psychology, Faculty of Behavioral Sciences, Saarland University, Building A1.7, D-66123 Saarbrücken, Germany
2013). Typical effects in selective attention paradigms seem to be sensitive to the proximity of the hands to the stimulus display (for a review, see Tseng, Bridgeman, \& Juan, 2012). For example, greater accuracy and shorter reaction times have been found for stimuli close to the hands in simple discrimination tasks (Whiteley, Kennett, Taylor-Clarke, \& Haggard, 2004). This has been demonstrated both for the hand making the task response and a hand passively placed near the stimuli (Reed, Grubb, \& Steele, 2006).

Other studies suggest that attentional disengagement is hampered when stimuli are in close proximity to the hands (Abrams, Davoli, Du, Knapp, \& Paull, 2008; Tseng et al., 2012). More precisely, Abrams et al. (2008) found steeper slopes in a visual search task, a more strongly pronounced attentional blink in a rapid serial visual presentation task, and a lack of inhibition of return in a Posner cueing task when the hand was proximal to the stimulus display rather than distant. Furthermore, switching between global and local scopes of attention is impeded near the hands: Davoli, Brockmole, Feng, \& Abrams (2012) demonstrated that the switch costs associated with switching between categorization of the overall shape of an object and categorization of its components (relative to task repetition) were increased when participants' hands were near the display (but see Weidler \& Abrams, 2014, Exp. 2, showing a reduction of task switch costs near the hands). These findings indicate that the attentional processing of both task-relevant and task-irrelevant information can be enhanced if presented near the hands.

In a recent study, Weidler \& Abrams (2014) found that the Eriksen flanker effect (Eriksen \& Eriksen, 1974) was eliminated near the hands. In the Eriksen flanker task, participants are instructed to respond to the identity of a target letter in a fixed location (typically the center of the screen) while ignoring distractor letters surrounding it. The distractors can be associated with the same or a different response to the target. 
If target and distractor response are in conflict, larger response times and/or error rates are typically observed. The authors argued that response conflict was reduced near the hands as a result of enhanced cognitive control. This result merits further examination. First the only other study to explore a response interference effect depending on hand proximity is that of Davoli et al. (2010), who found a reduced Stroop interference near the hands. ${ }^{1}$ As acknowledged by Weidler \& Abrams (2014), however, this result can be alternatively interpreted from a different perspective (as Davoli et al. did), namely in terms of a reduction of semantic processing near the hands. In other words, the study by Davoli et al. lends no unambiguous support for the hand proximal reduction of response conflict. Most importantly, in light of the other findings using attentional paradigms described above, the elimination of the Eriksen flanker effect, a robust and well-established response conflict phenomenon, seems surprising. As mentioned, attentional processing of visual stimuli appears to be enhanced when the stimuli are presented close to the hands. As evidenced by several findings, attentional disengagement in particular seems to be hampered (Abrams et al., 2008; Tseng et al., 2012; but see also Schultheis \& Carlson, 2013, indicating that the phenomenon might be more complex). It is unclear why the visual processing of distractors should interfere less with the task when they are presented close to the hands. From the findings concerning hampered disengagement, one might even predict an increased flanker effect near the hands unless there is either another mechanism reducing conflict interference - potentially enhanced cognitive control as proposed by Weidler \& Abrams (2014) - or otherwise participants manage to avoid distractor processing altogether, suggesting an alternative explanation in terms of narrowed spatial attention.

It is of course possible that more stimulus-driven, involuntary attentional components are affected differently by hand proximity than more goal-driven, controlled components, which are elicited following the detection of response conflict in a congruency task (Petersen \& Posner, 2012). In this regard, it is important to consider another phenomenon that is typically found in response interference paradigms in general and in the flanker task in particular, namely the Gratton or Congruency Sequence effect (CSE; Gratton, Coles, \& Donchin, 1992; for a recent review, see Duthoo, Abrahamse, Braem, Boehler, \& Notebaert, 2014). The CSE refers to the phenomenon that response conflict effects are usually smaller following an incompatible trial than following a compatible

\footnotetext{
${ }^{1}$ There are two other studies exploring the influence of hand posture on the flanker effect. Davoli \& Brockmole (2012) and Murchison \& Proctor (2015) found that positioning the hands between target and distractor reduced the flanker effect, suggesting that the hands may be used as a visual anchor, shielding spatial attention from outside influence. Although related, this is a research question different from the one posed by Weidler and Abrams (see also Weidler \& Abrams, 2014, p.465).
}

trial. The effect typically occurs in the Stroop task, the flanker task, and the Simon task (Egner, 2007), but also in the evaluative priming paradigm (Frings \& Wentura, 2008; Greenwald, Draine, \& Abrams, 1996).

There is extensive debate on the mechanism underlying the CSE. Interestingly, the CSE is often explained in terms of an up-regulation of cognitive control in response to conflict detection, with a relative enhancement of target processing and a relative impairment of distractor processing following an incompatible trial (Botvinick, Braver, Barch, Carter, \& Cohen, 2001). Alternative accounts stress the importance of episodic memory and priming for the CSE (Egner, 2007; Hommel, Proctor, \& Vu, 2004). It seems fair to conclude that neither explanation on its own is sufficient to explain the CSE, and there seems to be a residual contribution to the CSE which is more clearly attributable to cognitive control (Kim \& Cho, 2014; Weissman, Jiang, \& Egner, 2014; for a discussion, see Duthoo et al., 2014). Thus, an effect of a hand proximity manipulation on the CSE does not provide conclusive evidence of altered cognitive control. Conversely, however, it is plausible to assume that altered cognitive control - as hypothesized by Weidler \& Abrams (2014) - would not leave the CSE unaffected.

Thus, to conclude, whereas it remains unclear what precise influence of hand proximity would be predicted in the case of the flanker effect itself, we can assume that if hand proximity elicits an adaption in cognitive control, this should have measurable effects on the CSE. Since the CSE - if interpreted in terms of cognitive control - would reflect inter-trial fluctuations of cognitive control, a permanent increase of cognitive control - as proposed for the hand proximal region - should diminish these fluctuations.

Using a nearby hands set-up and an Eriksen flanker task, we investigated the effect of hand proximity on response conflict and on congruency sequence effects. We employed a standard version of the Eriksen flanker task, which we combined with a manipulation that allowed the task to be carried out with the hands either close to or relatively far away from the stimuli (see Fig. 1). We used a within-subjects design, counterbalancing the order of near and far conditions across participants.

\section{Methods}

\section{Participants}

Thirty-two students of Saarland University (22 female, ten male, aged 19-32 years, median age $=23$ ) took part in the experiment in exchange for a payment of 4 Euros. Given $N=$ 32 and $\alpha=.05$, effects of size $d_{Z}=0.51$ (i.e., medium-sized effects; Cohen, 1988) could be detected with a probability of 1 $-\beta=.80$ (G*Power; Faul, Erdfelder, Lang, \& Buchner, 


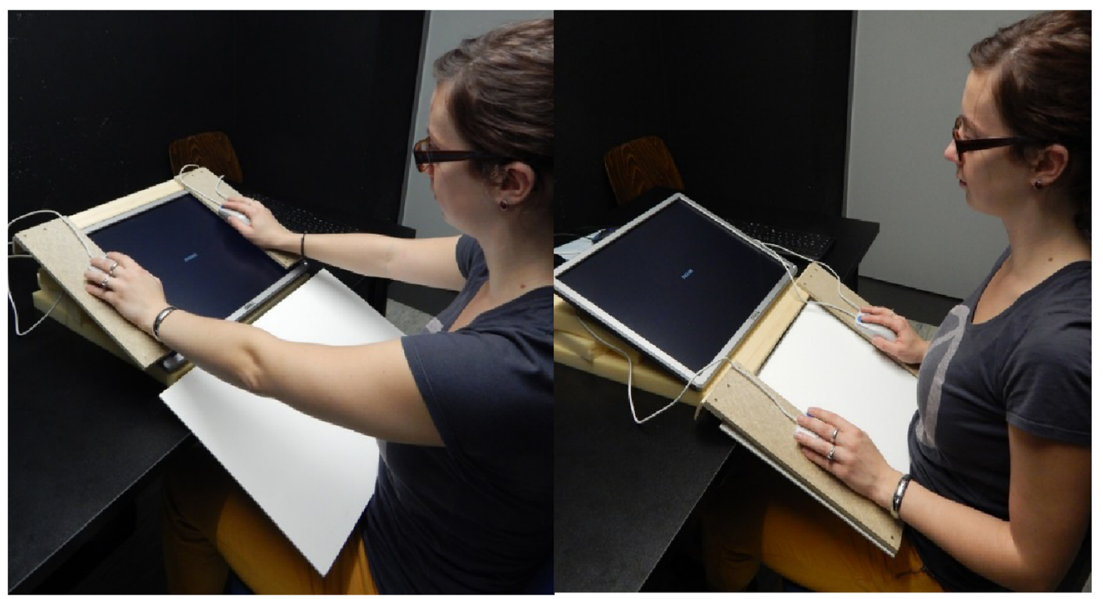

Fig. 1 Photographic depiction of the experimental set-up for the near (a) and far (b) condition, respectively. Participants placed their hands next to the screen in the near condition and on their laps in the far condition, while visual distance to the display remained constant

2007). Test power was $1-\beta=.95$ for effects of size $d_{z}=0.66$ (i.e., effects in between medium and large according to Cohen, 1988). Note that Weidler \& Abrams (2014) reported a 2 (compatibility) $\times 2$ (hand distance) interaction effect with error rates as the dependent variable of $d_{z}=0.88$.

\section{Apparatus}

Participants were seated $45 \mathrm{~cm}$ from a 19 -in. flat screen lying near-horizontally on a desk in a dimly lit room. The set-up is depicted in Fig. 1. Two computer mice were fixed to the left and right side of a wooden frame to serve as input devices. Target responses were made by pressing either a button on the left mouse or a button on the right mouse. In the "far" condition, the frame covered a wooden board which had the same size as the computer screen and was placed on the participants' lap. In this condition, the distance between the participants' hands and the stimuli was approximately $33 \mathrm{~cm}$. In the "near" condition, the frame was placed on top of the computer screen such that the participants' hands were placed close to the sides of the display. In this condition, the distance between each hand and the stimuli was approximately $12 \mathrm{~cm}$. The wooden board was placed on the participants' lap in this condition as well, to keep the participants' distance from the screen constant across conditions.

\section{Materials}

A standard flanker task (Eriksen \& Eriksen, 1974) was used. Participants had to respond to four different letters $(\mathrm{C}, \mathrm{H}, \mathrm{S}$, and $\mathrm{K}$ ) with one of two different responses (left or right). Half of the participants responded to $\mathrm{C}$ and $\mathrm{H}$ with the left button and to $\mathrm{S}$ and $\mathrm{K}$ with the right button. For the other half of participants, this response setting was reversed. The target was always presented in the center of the screen and flanked on either side by two letters that came from the same letter set (i.e., C, H, S, or K). The four flanking letters were always identical to each other and participants were explicitly instructed to ignore them. Letters were presented in Courier New font, size 10 (white on black background). The letter string was approximately $4 \mathrm{~cm}$ in width (subtending a visual angle of approx. 5.1 ${ }^{\circ}$ ) and $1 \mathrm{~cm}$ in height (approx. 1.3 $3^{\circ}$.

\section{Procedure}

At the beginning of each trial, a central fixation cross was presented for $250 \mathrm{~ms}$. This was followed by a string of five letters, with the target letter always appearing in the same location as the fixation cross. If an incorrect response was made, the message "Wrong!" was presented in red for 2, $000 \mathrm{~ms}$. If no response to the target letter was made within $1,200 \mathrm{~ms}$, the message "Unfortunately, you were too slow!" was presented for $2,000 \mathrm{~ms}$. After either this feedback or after a correct response, the screen went black for $1,000 \mathrm{~ms}$ before the next trial began.

Before the two experimental blocks (i.e., near and far conditions), participants worked through 16 practice trials, which also provided feedback on correct responses. The practice trials always used the same distance condition (near or far) as the first experimental block.

Each block consisted of 64 trials (i.e., 128 trials total). On half the trials, the target and distractor response were compatible with each other, and on the other half of trials, they were incompatible. Every possible combination of target and distractor was drawn randomly and repeated four times during each block. This led to an expected 63 trials with a compatible predecessor trial and an expected 63 trials with an incompatible predecessor trial. 


\section{Results}

Reaction times below $250 \mathrm{~ms}$ were excluded from the analyses, as were reaction times above the response deadline of 1 , $200 \mathrm{~ms}$. This led to the exclusion of a total of 30 trials $(0.7 \%$ of trials). We also excluded trials with erroneous responses from the reaction time analyses. This led to the exclusion of 262 trials ( $6.5 \%$ of trials). For the analyses of the CSE, the first trial of each block was excluded as well. Due to randomized presentation and these exclusion criteria, our analyses of the CSE is based on a total of 1,999 trials $(\mathrm{M}=62.7$ per participant) with a compatible predecessor trial and 2,006 trials $(\mathrm{M}=62.5$ per participant) with an in compatible predecessor trial.

\section{Flanker effect}

Mean response times (RTs) for the conditions of interest are shown in Table 1. As can be seen, numerically the flanker effect (for reaction times) was indeed smaller in the hands near condition compared to the hands far condition. We conducted a $2 \times 2$ repeated measures ANOVA with the factors response compatibility (compatible vs. incompatible) and hand proximity (near vs. far) and with latency of correct responses as the dependent measure. There was a main effect of compatibility, $F(1,31)=24.01, p<.001, d_{z}=.87$, with participants responding significantly slower when distractors were incompatible with the target response than when they were compatible. Neither the main effect of hand proximity nor the interaction between compatibility and hand proximity approached significance, both $F \mathrm{~s}<1$. Separate $t$-tests for the near and far conditions showed a significant Eriksen flanker effect in both

Table 1 Mean response times (in ms) and mean accuracy (in \%) as a function of hand proximity and flanker-target compatibility (standard deviations in parentheses)

\begin{tabular}{lll}
\hline & Hands & \\
\hline $\begin{array}{l}\text { Trial type } \\
\text { Response times }\end{array}$ & Near & Far \\
$\quad$ Compatible & $568(68)$ & $567(66)$ \\
Incompatible & $589(72)$ & $593(72)$ \\
$\quad$ Flanker effect & $21(34)$ & $26(37)$ \\
Accuracy & & \\
Compatible & $.96(.04)$ & $.95(.07)$ \\
Incompatible & $.94(.06)$ & $.92(.05)$ \\
Flanker effect & $.03(.06)$ & $.03(.08)$ \\
\hline
\end{tabular}

Note. The flanker effect is computed as the difference in response times and accuracy between compatible trials and incompatible trials. A positive difference indicates better performance in compatible trials. Discrepancies between the value of the Flanker effect and the difference of the means depicted are due to rounding the near, $t(31)=3.48, p=.002, d_{z}=.62$, and the far condition, $t(31)=4.04, p<.001, d_{z}=.71$.

The same pattern emerged for the error rates (see Table 1). The main effect of compatibility reached significance, $F(1,31)$ $=8.51, p=.007, d_{z}=.52$, with participants committing more errors if the distractor response was incompatible with the target response than when they were compatible. Neither the main effect of hand proximity nor the interaction between compatibility and hand proximity approached significance, both $F \mathrm{~s}<1$.Separate $t$-tests for the near and far conditions showed a significant Eriksen flanker effect in both the near, $t(31)=2.97, p=.005, d_{z}=.53$, and the far condition, $t(31)=$ $1.96, p=.030$ (one-tailed), $d_{z}=.35$.

\section{Congruency sequence effects}

Mean RTs for the conditions of interest are shown in Table 2. In the far condition, a $2 \times 2$ ANOVA with the factors compatibility in the previous trial, compatibility in the current trial, and reaction times as the dependent measure revealed a significant main effect of compatibility in the previous trial, $F(1$, $31)=5.70, p=.023, d_{z}=.42$, a significant main effect of compatibility in the current trial, $F(1,31)=15.92, p<.001$, $d_{z}=.71$, as well as a significant interaction between compatibility in the previous trial and compatibility in the current trial (i.e., a congruency sequence effect), $F(1,31)=6.69, p=.015$, corresponding to a CSE of $M=28 \mathrm{~ms}\left(S D=62 \mathrm{~ms} ; d_{z}=.46\right)$.

In the near condition, the corresponding analysis revealed a significant main effect of compatibility in the previous trial, $F(1,31)=5.25, p=.029, d_{z}=.40$, a significant main effect of compatibility in the current trial, $F(1,31)=12.84, p=.001, d_{z}$ $=.63$, but no hint of an interaction between compatibility in the previous trial and compatibility in the current trial (i.e., no significant congruency sequence effect), $F<1$. The CSE was $M=-5 \mathrm{~ms}\left(S D=79 \mathrm{~ms} ; d_{z}=.07\right)$.

The CSE in the near condition was thus significantly smaller than the CSE in the far condition, $t(17)=2.61, p=.018 .^{2}$

In anticipation of the discussion, we conducted a further analysis by taking the four flanker difference variables (i.e., RT incongruent minus RT congruent for flanker far, preceding trial compatible; flanker far, preceding trial incompatible; flanker near, preceding trial compatible; flanker near, preceding trial incompatible) as conditions of one within-participants factor. From the theoretical perspective of a control approach, the following orthogonal contrasts are meaningful: The first Helmert contrast (i.e., flanker far, preceding trial compatible, vs. the average of the three remaining conditions) contrasts the single condition without control efforts with conditions that are either associated with temporarily increased control (i.e.,

\footnotetext{
${ }^{2}$ A t-test for trimmed means (see Wilcox, 1997, 1998) with a trimming of $\gamma=.20$ was used to adequately account for outlying values (at both tails) in the near condition.
} 
Table 2 Mean reaction times (in $\mathrm{ms}$ ) and accuracy rates as a function of compatibility in trials $n-1$ and $n$ in the near-hands condition. Standard deviation in parentheses

\begin{tabular}{|c|c|c|c|}
\hline Current trial & Compatible & Incompatible & $\begin{array}{l}\text { Flanker } \\
\text { effect }\end{array}$ \\
\hline \multicolumn{4}{|l|}{ (a) Response times } \\
\hline \multicolumn{4}{|l|}{ Near hands } \\
\hline Previous compatible & $563(72)$ & $584(78)$ & $21(58)$ \\
\hline Previous incompatible & $574(72)$ & $595(74)$ & $21(43)$ \\
\hline $\begin{array}{l}\text { Congruency sequence effect } \\
\text { (CSE) }\end{array}$ & & & $-1(79)$ \\
\hline Far hands & & & \\
\hline Previous compatible & $553(66)$ & $593(71)$ & $40(52)$ \\
\hline Previous incompatible & $581(77)$ & $593(76)$ & $12(44)$ \\
\hline $\begin{array}{l}\text { Congruency sequence effect } \\
\text { (CSE) } \\
\text { (b) Accuracy }\end{array}$ & & & $28(62)$ \\
\hline \multicolumn{4}{|l|}{ Near hands } \\
\hline Previous compatible & $.97(.05)$ & $.93(.08)$ & $.04(.09)$ \\
\hline Previous incompatible & $.95(.06)$ & $.93(.07)$ & $.02(.07)$ \\
\hline $\begin{array}{l}\text { Congruency sequence effect } \\
\text { (CSE) }\end{array}$ & & & $.02(.12)$ \\
\hline \multicolumn{4}{|l|}{ Far hands } \\
\hline Previous compatible & $.94(.07)$ & $.92(.08)$ & $.02(.10)$ \\
\hline Previous incompatible & $.95(.08)$ & $.93(.07)$ & $.03(.11)$ \\
\hline $\begin{array}{l}\text { Congruency sequence effect } \\
\text { (CSE) }\end{array}$ & & & $-.00(.13)$ \\
\hline
\end{tabular}

Note. Discrepancies between effects and the difference of the corresponding means depicted are due to rounding

flanker far, preceding trial incompatible) or permanently increased control (i.e., the near-hands conditions); this contrast is significant, $F(1,31)=5.31, p=.028, d_{z}=.41$. The second Helmert contrast (i.e., flanker far, preceding trial incompatible, versus the average of the near conditions) contrasts the condition with temporarily increased control to those with permanently increased control; this contrast is insignificant, $F(1,31)=1.12, p=.298, d_{z}=.19$. The third Helmert contrast is the already known test for the CSE effect within the near condition (see above).

Corresponding analyses for accuracy only revealed the known main effects of compatibility in the current trial (see above), all other $F_{\mathrm{s}}<1.46$, ns.

\section{Discussion}

We found that hand stimulus distance had no significant effects on the magnitude of the flanker effect itself. Both in the "near" and the "far" condition, clear flanker effects of comparable size emerged, in both the reaction times and the accuracy rates. However, we did find a modulation of the congruency sequence effect (CSE; Gratton et al., 1992). In the far condition, we clearly replicated the well-known CSE, that is, the flanker effect is significantly reduced if the preceding trial was an incompatible trial compared to a compatible trial. In the near condition, however, this effect was completely eliminated.

Thus, on the one hand, with our set-up, we found no support for a modulation of the Eriksen flanker effect corresponding to the interaction found by Weidler \& Abrams (2014, Exp. 1). On the other hand, we found evidence of CSE modulation that is compatible with Weidler and Abrams' general notion that is, enhanced cognitive control near the hands. In the following, we will separately discuss both issues.

Several parameters in our set-up differed from the one used by Weidler \& Abrams (2014; Exp. 1): We used (a) a somewhat larger response deadline, (b) a smaller visual angle, and (c) a somewhat different set-up to realize the near versus far condition. The response deadline (i.e., 1,200 $\mathrm{ms}$ instead of 1, $000 \mathrm{~ms})^{3}$ seems to be the least plausible candidate for explaining the difference. Nevertheless, we should recall here that the moderation of the flanker effect found by Weidler and Abrams was in the accuracy data. Thus, subtle changes that might lead to a reduction in errors might have changed the effects as well.

A potentially better explanation for our failure to obtain a modulation of the flanker effect itself pertains to the difference in visual angle of the stimulus presentation. Distractors presented close to the center of attention have been shown to interfere more strongly with the target response under some conditions (Beck \& Lavie, 2005; Eriksen \& Eriksen, 1974), indicating that they are more likely to be processed. Since attentional disengagement seems to be hampered near the hands, thereby potentially increasing reaction time costs caused by task-irrelevant stimuli (Abrams et al., 2008; Tseng et al., 2012; Weidler \& Abrams, 2013), this difference in visual angle may have led to an initial engagement of attention in our, but not in Weidler \& Abrams' (2014) near condition. If our smaller stimulus presentation format indeed made participants more likely to engage the distractors compared to the format used by Weidler \& Abrams (2014), it is possible that the cost of delayed disengagement counteracted any reaction time benefit conveyed by enhanced cognitive control. This possibility of mutually interfering processes near the hands also underscores the usefulness of the CSE as an alternative measure of cognitive control.

Finally and most importantly, the posture manipulation we employed was different from the one used by Weidler \& Abrams (2014). The near-hands condition employed by the authors was realized by two buttons that were attached to the center of each side of an upright monitor; thus, arms were raised and the palms were directed towards the stimuli, whereas in the far condition, participants held their hands flat on

\footnotetext{
${ }^{3}$ The increase seemed warranted to us due to piloting testing.
} 
their lap (see Abrams et al., 2008, for an illustration). In our setup the hands lay on the same straight line in both near and far conditions; thus, arms were not raised to the same extent as in the study by Weidler and Abrams and palms were not directed towards the stimuli (see Fig. 1).

With regard to the raised hands, Weidler \& Abrams (2013) already tested whether this confound might be responsible for effect moderations that are typically interpreted as moderations by distance. In their set-up, the typical confound (i.e., raised hands in the near condition but not in the far condition) was switched by realizing a far-hands condition with raised hands. They found the same near-by hands moderation of a visual search effect as Abrams et al. (2008, who had used the manipulation with raised hands in the near condition).

Thus, the discussion should focus on the fact that in our setup the palms were not directly facing the stimuli. In this regard, it is interesting to note that Reed, Betz, Garza, \& Roberts (2010) explored the role of stimulus-directed versus stimulus-averted palm in a spatial cueing paradigm with target stimuli randomly presented either to the left or right of the screen's center. They found decreased target detection latencies if the participant's palm was located near and towards the target position (e.g., the left hand is positioned slightly to the left of the left target position, with fingers tipping at the display) in comparison to targets that appeared on the other side of the display. A comparable effect was not found if the palm was near but averted from the target (e.g., the left hand is positioned between the left target position and the centered fixation cross). Thus, it might turn out in future research that at least part of so-called "near-by hands" effects is in fact a "potential-to-grasp" effect (see also Thomas, 2015). ${ }^{4}$ However, it is important to note that in our setup the palms were not averted from stimuli: Hands were directed towards the plane in which the stimuli are presented and a simple sideways movement would have enabled participants to grasp them. It is not prima facie apparent why this posture should constitute an insufficient nearby hands condition.

Nevertheless, we have found an effect of our distance manipulation. In line with Weidler \& Abrams' (2014) account of altered cognitive control near the hands, we found that hand proximity modulated the congruency sequence effect. A classical explanation for the CSE has been in terms of cognitive control (Botvinick et al., 2001). If cognitive control is indeed (permanently) enhanced near the hands, it would be possible that there is no additional benefit resulting from conflict detection.

However, even if we tentatively accept this hypothesis, at least at first sight the question remains why the overall flanker effect is not reduced in the near-hands condition as compared to the far-hands condition, due to permanently larger control efforts. A possible response is given by the results of the

\footnotetext{
${ }^{4}$ We thank Richard A. Abrams for providing us with this argument.
}

additional analysis reported above, which shows that the effects in fact do not necessarily violate assumptions of a control approach: There is a significant difference in the flanker effects between the single condition for which reduced control is hypothesized (i.e., far hands, preceding trial compatible) and the conditions for which either temporarily (i.e., far hands, preceding trial incompatible) or permanently increased control (i.e., near hands) is hypothesized. And there is no significant difference in the flanker effects between those conditions for which either temporarily or permanently increased control is hypothesized. Of course, this is the maximally benevolent interpretation of the data from the viewpoint of a control approach. But we should point out that Weidler and Abram's (2014) interpretation of enhanced cognitive control does not rest solely on their flanker experiment. It is also compatible with the reduction of task switching costs, found in their Experiment 2, and the reduction in Stroop interference, found by Davoli and colleagues (2010). Nevertheless, with regard to the interpretation of our results, two possible objections have to be considered.

The first possible objection would be further insistence on our failure to find a moderation of the overall flanker effect by hand distance in light of the large moderation found by Weidler \& Abrams (2014). It seems as though - even if enhanced cognitive control near the hands tends to decrease the flanker effect - a counter process " $\mathrm{x}$ " has to be considered that causes an increase in the flanker effect near the hands. In the introduction, we gave one suggestion. In visual search experiments, Abrams et al. (2008) as well as Weidler \& Abrams (2013) found steeper slopes in the near-hands condition and interpreted this result in terms of increased difficulties to disengage attention from distractors. In this regard, one might assume that flankers receive more processing (due to disengagement problems) in the near condition as compared to the far condition.

The second objection concerns the fact that the explanation of the CSE in terms of cognitive control is disputed. Alternative accounts have been proposed, stressing the role of episodic memory and feature integration for the CSE (Duthoo et al., 2014; Egner, 2007; Hommel et al., 2004). Further accounts interpret the CSE as reflecting expectations for the following trial (Duthoo et al., 2014; Egner, 2007). It is thus possible that the modulation of the CSE that we have found reflects a mechanism other than an adaptation in cognitive control. Thus, our result merits closer investigation and further corroboration. To exclude an explanation in terms of feature integration, more complex set-ups using a larger set of stimuli and response mappings should be employed (Kim \& Cho, 2014; Weissman et al., 2014). If, however, it turns out that the moderation of the CSE by hand distance should be better conceptualized as a moderation of feature integration processes than a moderation of cognitive control, the nearby-hands research will be enriched by a further stimulating topic. 
Research on nearby hands has yielded a host of fascinating and seemingly heterogeneous findings which pertain to a number of cognitive mechanisms, such as delayed disengagement, shallower semantic processing, faster engagement, or enhanced cognitive control. It is our belief the field stands only to benefit from an integration of these results into a shared theoretical framework, taking into account different components of attention, characteristics of the tasks, and characteristics of the posture manipulation in order to achieve more precise predictions across differing experimental setups. Employing a measure such as the CSE as a complementary index of cognitive control can provide valuable information when the hypothesized effects are not evident on the level of the individual trial.

\section{References}

Abrams, R. A., Davoli, C. C., Du, F., Knapp, W. K., \& Paull, D. (2008). Altered vision near the hands. Cognition, 107, 1035-1047.

Beck, D. M., \& Lavie, N. (2005). Look here but ignore what you see: Effects of distractor at fixation. J Exp Psychol Hum Percept Perform, 31, 592-607.

Botvinick, M. M., Braver, T. S., Barch, D. M., Carter, C. S., \& Cohen, J. D. (2001). Conflict monitoring and cognitive control. Psychol Rev, 108, 624-652.

Brockmole, J. R., Davoli, C. C., Abrams, R. A., \& Witt, J. K. (2013). The world within reach: Effects of hand posture and tool-use on visual cognition. Current Directions in Psychological Science, 22, 38-44.

Cohen, J. (1988). Statistical power analysis for the behavioral sciences (2nd ed.). Hillsdale: Lawrence Erlbaum Associates.

Davoli, C. C., \& Brockmole, J. R. (2012). The hands shield attention from visual interference. Atten Percept Psychophys, 74, 1386-1390.

Davoli, C. C., Brockmole, J. R., Feng, D., \& Abrams, R. A. (2012). Switching between global and local scopes of attention is resisted near the hands. Visual Cognition, 20, 659-668.

Davoli, C. C., Du, F., Montana, J., Garverick, S., \& Abrams, R. A. (2010). When meaning matters, look but don't touch: The effects of posture on reading. Mem Cognit, 38, 555-562.

Duthoo, W., Abrahamse, E. L., Braem, S., Boehler, C. N., \& Notebaert, W. (2014). The heterogeneous world of congruency sequence effects: An update. Frontiers in Psychology, 5, 1-7.

Egner, T. (2007). Congruency sequence effects and cognitive control. Cognitive, Affective, and Behavioral Neuroscience, 7, 380-390.

Eriksen, B. A., \& Eriksen, C. W. (1974). Effects of noise letters upon the identification of a target letter in a nonsearch task. Percept Psychophys, 16, 143-149.
Faul, F., Erdfelder, E., Lang, A.-G., \& Buchner, A. (2007). G*Power 3: A flexible statistical power analysis program for the social, behavioral, and biomedical sciences. Behav Res Methods, 39, 175-191.

Frings, C., \& Wentura, D. (2008). Trial-by-trial effects in the affective priming paradigm. Acta Psychol (Amst), 128, 318-323.

Gratton, G., Coles, M. G. H., \& Donchin, E. (1992). Optimizing the use of information: Strategic control of activation of responses. $J$ Exp Psychol Gen, 121, 480-506.

Greenwald, A. G., Draine, S. C., \& Abrams, R. L. (1996). Three cognitive markers of unconscious semantic activation. Science, 273, 1699-1702.

Hommel, B., Proctor, R. W., \& Vu, K.-P. L. (2004). A featureintegration account of sequential effects in the Simon task. Psychol Res, 68, 1-17.

Kim, S., \& Cho, Y. S. (2014). Congruency sequence effect without feature integration and contingency learning. Acta Psychol (Amst), 149, $60-68$.

Murchison, N. M., \& Proctor, R. W. (2015). How hand placement modulates interference from extraneous stimuli. Atten Percept Psychophys, 77, 340-352.

Petersen, S. E., \& Posner, M. I. (2012). The attention system of the human brain: 20 years after. Anпи Rev Neurosci, 35, 73-89.

Reed, C. L., Betz, R., Garza, J. P., \& Roberts, R. J. (2010). Grab it! Biased attention in functional hand and tool space. Attention, Perception and Psychophysics, 72, 236-245.

Reed, C. L., Grubb, J. D., \& Steele, C. (2006). Hands up: Attentional prioritization of space near the hand. J Exp Psychol Hum Percept Perform, 32, 166-177.

Schultheis, H., \& Carlson, L. A. (2013). Determinants of attentional modulation near the hands. Frontiers in Psychology, 4, 858.

Thomas, L. E. (2015). Grasp posture alters visual processing biases near the hands. Psychol Sci, 26, 625-632.

Tseng, P., Bridgeman, B., \& Juan, C. H. (2012). Take the matter into your own hands: A brief review of the effect of nearby-hands on visual processing. Vision Res, 72, 74-77.

Weidler, B. J., \& Abrams, R. A. (2013). Hand proximity — not arm posture-alters vision near the hands. Atten Percept Psychophys, 75, 650-653.

Weidler, B. J., \& Abrams, R. (2014). Enhanced cognitive control near the hands. Psychon Bull Rev, 21, 462-469.

Weissman, D. H., Jiang, J., \& Egner, T. (2014). Determinants of congruency sequence effects without learning and memory confounds. $J$ Exp Psychol Hum Percept Perform, 40, 2022-2037.

Whiteley, L., Kennett, S., Taylor-Clarke, M., \& Haggard, P. (2004). Facilitated processing of visual stimuli associated with the body. Perception, 33, 307-314.

Wilcox, R. R. (1997). Introduction to robust estimation and hypothesis testing. San Diego: Academic.

Wilcox, R. R. (1998). How many discoveries have been lost by ignoring modern statistical methods? American Psychologist, 53, 300-314. 